\title{
3 Verbesserungsideen erfassen und nutzen: Betriebliches Vorschlagswesen und Beschwerdemanagement
}

Christine Kuch

Betriebliches Vorschlagswesen und Beschwerdemanagement sind aus Sicht des Qualitätsmanagements zwei Möglichkeiten, Ideen für Verbesserung zu generieren. Das betriebliche Vorschlagswesen richtet sich vor allem an Mitarbeiter, das Beschwerdemanagement vor allem an Patienten. Auf beide Verfahren wird nachstehend kurz eingegangen. Zunächst jedoch erfolgt an dieser Stelle in Tabelle 10 eine Übersicht, wie beide Verfahren entwickelt und eingerichtet werden können.

\subsection{Betriebliches Vorschlagswesen}

Das Betriebliche Vorschlagswesen zielt darauf, das Ideenpotenzial auch von Mitarbeitern der Basis und nicht nur von Leitungskräften einer Organisation zu nutzen. Im Rahmen des betrieblichen Vorschlagswesens können Mitarbeiter Verbesserungsvorschläge einreichen und erhalten dafür Belohnungen, die sowohl in Form von Geld- als auch Sachprämien erbracht werden. Man unterscheidet ein strukturiertes und ein unstrukturiertes Vorgehen für das betriebliche Vorschlagswesen.

Damit wird deutlich, dass das Betriebliche Vorschlagswesen eine Methode ist, die sich für größere Organisationen eignet und bei kleineren Organisationen, zu denen in aller Regel die Arztpraxis gehört, in der strukturierten Form wenig hilfreich ist. In einer Arztpraxis können einige Prinzipien des Betrieblichen Vorschlagwesens übernommen werden. Von einer individuellen Prämie, wie sie normalerweise im betrieblichen Vorschlagwesen vorgesehen ist, 
Tab. 10 Vorgehen für die Entwicklung von Beschwerdemanagement oder betrieblichem Vorschlagswesen

\begin{tabular}{|c|c|c|}
\hline Inhalt & $\begin{array}{l}\text { Beteiligte/Verant- } \\
\text { wortlich }\end{array}$ & $\begin{array}{l}\text { Zeitaufwand } \\
\text { (maximal) }\end{array}$ \\
\hline $\begin{array}{l}\text { 1. Zielsetzung klären: Was möchte man mit einem } \\
\text { Beschwerdemanagement erreichen? Was soll der konkrete } \\
\text { Nutzen sein? ODER Was möchte man mit einem Vorschlags- } \\
\text { wesen erreichen? Was soll der konkrete Nutzen sein? }\end{array}$ & $\begin{array}{l}\text { Praxisleitung/ } \\
\text { Vorstand, ggf. } \\
\text { QM-Beauftragte }\end{array}$ & $\begin{array}{l}\text { Tag } 0 \\
1 . \text { Sitzung à } \\
\text { max. } \\
1,5 \text { Stunden }\end{array}$ \\
\hline $\begin{array}{l}\text { 2. Verfahren entwickeln: Wie können Beschwerden erfasst } \\
\text { werden? Beim Beschwerdemanagement zusätzlich: Wie } \\
\text { soll konkret auf eine akute Beschwerde reagiert werden? } \\
\text { Oder: Wie können Vorschläge eingebracht werden? }\end{array}$ & Projektteam & $\begin{array}{l}1 \text { bis max. } \\
3 \text { Sitzungen }\end{array}$ \\
\hline $\begin{array}{l}\text { 3. Verantwortlichkeiten festlegen (Wer bekommt den Hut } \\
\text { dafür auf, dass das Verfahren umgesetzt wird? Wer ist für } \\
\text { welchen Schritt verantwortlich?) }\end{array}$ & Projektteam & \multirow[t]{3}{*}{$\begin{array}{l}1 \text { Sitzung für } \\
\text { Ablaufschrit- } \\
\text { te } 3,4 \text { und } 5\end{array}$} \\
\hline $\begin{array}{l}\text { 4. Umsetzung vorbereiten (Infomaterial erstellen, beim } \\
\text { Beschwerdemanagement ggf. Mitarbeiterschulung planen } \\
\text { etc.) und Evaluation planen (Wann müssen wir schauen, ob } \\
\text { das Verfahren gut funktioniert? Wer macht das wie?) }\end{array}$ & $\begin{array}{l}\text { Projektteam, } \\
\text { Beauftragte }\end{array}$ & \\
\hline $\begin{array}{l}\text { 5. Kommunikation planen: Wie erfahren Patienten vom } \\
\text { Beschwerdemanagement? ODER Wie erfahren Mitarbeiter } \\
\text { vom Beschwerdemanagement? Wer informiert wen wie } \\
\text { und wann? }\end{array}$ & Projektteam & \\
\hline $\begin{array}{l}\text { 6. Abschluss der Entwicklung, Beschluss und Start der } \\
\text { Umsetzung in einer Pilotphase }\end{array}$ & $\begin{array}{l}\text { Praxisleitung/ } \\
\text { Vorstand }\end{array}$ & \\
\hline $\begin{array}{l}\text { 7. Kommunikation des Vorhabens, ggf. Durchführung einer } \\
\text { Mitarbeiterschulung beim Beschwerdemanagement }\end{array}$ & $\begin{array}{l}\text { Praxisleitung/ } \\
\text { Vorstand, Beauftragte }\end{array}$ & \\
\hline $\begin{array}{l}\text { 8. Evaluation nach zuvor festgelegter Planung, ggf. } \\
\text { Überarbeitung/Verbesserung des Verfahrens }\end{array}$ & $\begin{array}{l}\text { QM-Beauftragte, ggf. } \\
\text { Projektteam }\end{array}$ & \\
\hline 9. Beschluss des neuen Verfahrens & $\begin{array}{l}\text { Praxisleitung bzw. } \\
\text { Vorstand }\end{array}$ & \\
\hline 10. Start der Routine & $\begin{array}{l}\text { Praxisleitung bzw. } \\
\text { Vorstand }\end{array}$ & \\
\hline
\end{tabular}

ist jedoch dringend abzuraten: Der entstehende Wettbewerb innerhalb der Gruppe ist für das Team schädlich, die Zusammenarbeit leidet darunter und Konflikte sind vorprogrammiert. Stattdessen können hier Prämien für das gesamte Team vorgesehen werden, die das Team als ganzes belohnen (und neben dem motivierenden Effekt eine Unterstützung des Zusammenhaltes innerhalb der Cruppe fördern).

Bei der strukturierten Form des betrieblichen Vorschlagswesens ist festgelegt, wie und auf welchem Wege Vorschläge formuliert und zur Bearbeitung und Ent- 
scheidung weitergeleitet werden. Zudem ist es für den Mitarbeiter auch ersichtlich, welche Prämie er für welchen Vorschlag, so er umgesetzt wird, erhalten kann: Meist werden festgelegte Prozentsätze beispielsweise der durch den Vorschlag eingesparten Kosten in Form von Sach- oder Geldprämien an die Mitarbeiter ausgeschüttet. Dieses Verfahren eignet sich insbesondere für große Organisationen, in denen der einzelne Mitarbeiter an der Basis keinen oder kaum Kontakt zu den Leitungskräften hat.

Bei der unstrukturierten Form des betrieblichen Vorschlagswesens können Mitarbeiter Vorschläge auf unterschiedlichste Art und Weise einbringen. Beispielsweise liegt ein Buch aus, in das man Ideen eintragen kann, im Computer existiert eine entsprechende Datei, ein Kasten ist aufgestellt, in den man Blätter mit schriftlichen Vorschlägen werfen kann. All diese Ideen werden in regelmäßigen „Ideen-Konferenzen“ gemeinsam besprochen und ggf. nach Beschluss der Leitung umgesetzt. Dieses Vorgehen ist für Arztpraxen im Allgemeinen besser geeignet, weil Verbesserungspotenziale schnell erkannt und genutzt werden können. Um die Idee der Belohnungen zu übernehmen, können zum Beispiel die Verbesserungsideen gezählt werden, und dann kann nach dem 50. gemachten Vorschlag oder nach dem 10. umgesetzten Vorschlag ein kleines Fest, ein Ausflug, ein gemeinsames Abendessen anstehen. Wichtig dabei ist, wie schon oben kurz angesprochen, dass Belohnungen in einer kleinen Organisation an die gesamte Gruppe gerichtet sein sollten, da individuelle Leistungsprämien einer guten Zusammenarbeit im Team abträglich sind.

\subsection{Beschwerdemanagement oder: Patientenbindung stärken}

Im Beschwerdemanagement wird beschrieben, wie in der Praxis mit Beschwerden von Kunden, in erster Linie mit Beschwerden von Patienten, umgegangen wird. Zudem wird geregelt, wie mit den gesammelten Verbesserungsvorschlägen zu verfahren ist.

Basis eines funktionierenden Beschwerdemanagements ist es, dass Beschwerden von Patienten (aber auch von allen anderen internen und externen Kundengruppen) ernst genommen und nicht als lästige Nörgelei abgetan werden. Lange Wartezeiten, Missverständnisse, unklare Angaben, Schmerzen all das kann dazu führen, dass Patienten ungehalten reagieren.

Die grundlegende Idee des Beschwerdemanagements ist es, dass diese Reaktionen Hinweise auf Qualitätsprobleme darstellen. Beschwerden sind insofern wertvolle Hinweise, die man „frei Haus“ erhält. Außerdem bieten sie Anlass, bei einem unzufriedenen Patienten wieder Zufriedenheit herzustellen und damit die Bindung des Patienten an die Praxis massiv zu erhöhen. Darüber hinaus ist es vorteilhaft, wenn Patienten ihre Beschwerde in der Praxis aussprechen, anstatt sie mit nach außen zu tragen und im Bekanntenkreis zu kommunizieren. Zumindest dort, wo Konkurrenz herrscht, ist jede Beschwerde, die nicht als Mundpropaganda die Runde macht, wertvoll. 
Ein weiteres Ziel des Beschwerdemanagements ist es, Mitarbeiter beim Umgang mit Beschwerden zu entlasten. In der Regel sind es die Mitarbeiter, die die Beschwerden „abbekommen“. Ein abgestimmtes Verfahren, das dem Mitarbeiter auch möglichst weitreichende Kompetenzen gibt, auf Beschwerden zu reagieren, gibt Sicherheit. Es hilft damit auch einem möglicherweise gereizten Mitarbeiter, ruhiger zu reagieren und damit erfolgreicher im Umgang mit der Beschwerde zu werden.

Das Beschwerdemanagement in einer Praxis umfasst:

- eine Vorgehensbeschreibung für den Umgang mit einer Beschwerde in der Praxis,

- eine Auswertung eingegangener Beschwerden und der darauf folgenden Konsequenzen (Beschwerdestatistik) und

- eine abgestimmte Strategie zum Umgang mit aktuellen Beschwerden im Gespräch.

\section{Vorgehensbeschreibung für den Umgang mit einer Beschwerde in der Praxis}

Für ein solches Vorgehen wird zum einen vereinbart, wie in der Praxis mit mündlichen bzw. mit schriftlichen Beschwerden umgegangen werden soll. Dabei können die folgenden Fragen beantwortet werden, nachdem man sich in die Rolle einer Person versetzt, die sich gerade beschwert:

- Wie werden die Beschwerden erfasst? Das schriftliche Aufnehmen einer Beschwerde unterstreicht die Relevanz, die man der Beschwerde zuschreibt. Zudem sichert eine einheitliche Aufnahme, dass keine relevanten Punkte vergessen werden.

- Gibt es unterschiedliche Gruppen von Beschwerden, mit denen unterschiedlich umgegangen werden sollte?

- Wann bekommt die Praxisleitung oder der Beschwerdebeauftragte die Beschwerde zur Entscheidung über das weitere Vorgehen vorgelegt?

- Welche Konsequenzen werden bei welchen Beschwerden ergriffen?

- Wie und wann erfährt der Beschwerdeführer von nachfolgenden Aktionen?

- Wie und von wem wird der Erfolg der ergriffenen Maßnahmen erfasst?

Zudem wird vereinbart, wie Mitarbeiter Beschwerden aufnehmen, die sie nur indirekt hören, etwa dadurch, dass sich zwei Patienten unterhalten.

\section{Auswertung eingegangener Beschwerden und der darauf folgenden Konsequenzen (Beschwerdestatistik)}

Es wird vereinbart, nach welchen Kriterien die Dokumentation der Beschwerden, mindestens einmal jährlich, von dem Qualitätsmanagementbeauftragten ausgewertet werden soll. Beispiele dafür sind:

Anzahl der Beschwerden, Art der Beschwerden (häufige Beschwerden?), Anteil erfolgreich bearbeiteter Beschwerden, Anteil von Patienten, die sich 
beschwert haben und weiterhin Patienten der Praxis sind, Art und Anzahl von auf Basis von Beschwerden erfolgreich umgesetzter Verbesserungen, Qualität des Umgangs mit Beschwerden aus Sicht der Mitarbeiter und aus Sicht der Praxisleitung usw.

Die Auswertung wird in einer Teambesprechung diskutiert und etwaige Konsequenzen werden vereinbart (z. B. Bearbeitung häufiger Beschwerden, Reflexion des Verhaltens der Praxisleitung).

\section{Strategie zum Umgang mit aktuellen Beschwerden im Gespräch}

Der Umgang mit Beschwerden stellt eine gewisse Herausforderung dar, da diese meistens dann erfolgen, wenn die eigene Belastung hoch ist. Daher sollte ein Vorgehen vereinbart werden, an das sich alle halten können. Deutlich bewährt hat sich das nachstehend beschriebene Vorgehen der „Drei lösungsorientierten Kniffe zum Umgang mit Beschwerden“ (Kuch 2008):

1. ausreden lassen und zuhören

- (falls notwendig) Konflikt/Missstimmung/Unzufriedenheit/... ansprechen(!)

- Beschwerdeführer ausreden lassen und: Zuhören! Wenn ein Beschwerdeführer zu einem späteren Zeitpunkt im Gespräch wieder anfängt, sich zu beschweren, heißt das nur, dass er vorher nicht vollständig ausgesprochen hat - einfach wieder abwarten, zuhören und von neuem starten!

2. Verantwortung übernehmen und nicht rechtfertigen

- entschuldigen (bei eigenem Verschulden), Verständnis äußern (das ist eine unangenehme Situation), Danken (Danke, dass Sie mir das sagen)

- nicht rechtfertigen, nicht auf der Sachebene argumentieren (Sparen Sie sich Begründungen („Wir haben gerade so viel zu tun.“, „Die andere Ärztin ist in Urlaub, ...“), sie heizen die Stimmung an dieser Stelle nur auf.)

3. Lösungsvorschläge erfragen und Umsetzung sicherstellen

- Lösungsvorschläge erfragen (In diesem Schritt übernimmt der Beschwerdeführer Verantwortung für die Lösung. Schlägt er unrealistische Lösungen vor, kann man darauf verweisen, dass man leider sofort zu dieser Lösung nicht in der Lage sei, die Idee aber aufnehmen und eine Umsetzung prüfen werde. Anschließend erfragt man wieder einen Lösungsvorschlag!)

- auf der Basis der Lösungsvorschläge des Beschwerdeführers konkrete Lösungen anbieten und deren Umsetzung vereinbaren

Kurz und knapp heißt das also: Den Beschwerdeführer ausreden lassen (vollständig, notfalls auch einfach mal bis 20 zählen), entschuldigen oder Verständnis äußern, danken für die Äußerung und sofort danach fragen, was aus Sicht des 
Beschwerdeführers eine gute Lösung wäre. Abschließend gemeinsam eine umsetzbare Lösung vereinbaren.

Die nachstehende Aufgabe soll den Leserinnen und Lesern die Möglichkeit geben, im Team dieses Vorgehen auszuprobieren

\section{Aufgabe 7 für anwendungsfreudige Leser}

„Umgang mit Beschwerden“

Bitte setzen Sie sich (jeweils) zu dritt zusammen. Klären Sie, wer:

- sich aktiv beschweren möchte,

- lösungsorientiert mit dieser Beschwerde umgehen möchte,

- den Ablauf des Gesprächs beobachten möchte, insbesondere in Hinblick auf die Beachtung der drei Kniffe!

Definieren Sie nun die Situation, in der einer der Beteiligten eine Beschwerde vorbringen möchte: Wie sieht die Situation aus? Wer sitzt/steht wo? Wer ist konkret beteiligt?

Stellen Sie sich bitte kurz auf die Situation ein, bevor Sie „loslegen“. Schlüpfen Sie ganz in Ihre Rolle! Beschwerdeführer: Sind Sie richtig sauer? Beschwerdeempfänger: Wollen Sie das Problem wirklich lösen? Beobachter: Wie sieht der erwartete Prozess aus?

Und dann geht es los, der Beschwerdeempfänger kann zur Unterstützung auch von einem Zettel mit den drei Kniffen „spicken“.

Anschließend bringt zunächst der Beschwerdeempfänger vor,

a) was ihm alles schon gut gelungen ist,

b) was er ggf. beim nächsten Mal ganz konkret anders machen möchte.

Dann gibt der Beschwerdeführer an,

a) was aus seiner Sicht gut gelungen ist und wie er sich dabei gefühlt hat,

b) was ihm beim nächsten Mal ganz konkret noch gut tun würde.

Abschließend können der oder die Beobachter noch bislang nicht genannte Ergänzungen abgeben,

a) was aus ihrer Sicht gut gelungen ist,

b) was beim nächsten Mal ganz konkret anders gemacht werden sollte.

\section{Literatur}

Kuch C: 3 lösungsorientierte Kniffe zum Umgang mit Beschwerden. Materialsammlung zur Vorkonferenz der SOLworld International Link-up. Köln 2008 